\title{
Des Transformations du Paysannat Africain à la Nouvelle Configuration des Rapports Villes-Campagnes au Cameroun
}

\author{
Ismaïla Datidjo, Anthropologue \\ Désiré Ndoki, Géographe \\ Claudin Karim Nana, Sociologue \\ Université de Dschang, Cameroun
}

Doi:10.19044/esj.2020.v16n17p133 URL:http://dx.doi.org/10.19044/esj.2020.v16n17p133

\section{Résumé}

Sans échapper à la force transformatrice qui affecte toutes les sociétés au monde, l'univers paysan africain et notamment celui camerounais est sans doute soumis à une transmutation qui lui confère un aspect nouveau. Il est transformé sous l'impulsion paysanne, de même que sous l'influence des mutations urbaines et par extension mondiales. Les répercussions de ces mutations se transposent dans les économies villageoises au point de marquer les relations que les paysans entretiennent avec les citadins. Héritier d'une tradition agricole ancienne à forte prédominance culturale léguée par les aïeuls, le paysannat africain en général et camerounais dans une moindre mesure, se présente aujourd'hui sous une forme alliant à la fois les acquis ancestraux et les faits relativement nouveaux issus des périodes coloniales et postcoloniales. Cet article se veut une réflexion portée sur le dynamisme que connait le monde paysan avec l'incursion coloniale et surtout l'accélération du processus de mondialisation qui évoque concomitamment continuité et rupture au sein des sociétés villageoises tiraillées entre traditions locales et innovations. Il s'agit d'appréhender la nouvelle identité du paysan camerounais soumis à la fois aux réalités d'une origine ancienne et à la modernité à partir des connexions villes-campagnes telles qu'elles se présentent aujourd'hui.

Mots-clés : Paysan, tradition, mutations sociales, villes-campagnes, modernité 


\title{
From the Transformations of the African Peasantry to the New Configuratio of Urban-Rural Relations in Cameroon
}

\author{
Ismaïla Datidjo, Anthropologue \\ Désiré Ndoki, Géographe \\ Claudin Karim Nana, Sociologue \\ Université de Dschang, Cameroun
}

\begin{abstract}
Without escaping the transforming force that affects all societies in the world, the African peasant world, and particularly that of Cameroon, is undoubtedly undergoing a transmutation which gives it a new look. It is transformed under the peasant impulse, as well as under the influence of urban and global changes. The repercussions of these changes are being transposed into village economies to the extent that they are having an impact on the relationship between farmers and city dwellers. Inheritor of an ancient agricultural tradition with strong cultural predominance bequeathed by the ancestors, the African peasantry in general and Cameroonian peasantry to a lesser extent, is today presented in a form that combines both ancestral achievements and relatively new developments from the colonial and postcolonial periods. This article aims to reflect on the dynamism that the peasant world is experiencing as a result of the colonial incursion and, above all, the acceleration of the globalization process, which at the same time evokes continuity and rupture within village societies torn between local traditions and innovations. It is a question of apprehending the new identity of the Cameroonian peasant subjected both to the realities of an ancient origin and to modernity from the urban-rural connections such as they appear today.
\end{abstract}

Keywords: Peasant, tradition, social changes, city-campaign, modernity

\section{Introduction}

Le monde se transforme au fur et à mesure que l'homme poursuit son dessein fondamental par l'entremise des progrès scientifiques et techniques qu'il opère au fil des générations. En conférant à l'humanité des conditions d'existence meilleures, le processus de transformation des sociétés a de tout temps influencé les modes de vie allant de ceux des groupes les plus restreints 
comme les familles, à ceux les plus complexes à l'image des sociétés urbaines en passant entre autres par les sociétés villageoises de dimensions relativement importantes. En Afrique, ces dernières sont elles aussi soumises à des transmutations qui témoignent de leur participation à la société globale au sein de laquelle il est quasi inconcevable d'admettre une partie qui soit totalement détachée du grand ensemble. Cela dit, les zones rurales en Afrique ne sont donc pas en marge du dynamisme qui caractérise l'essentiel des sociétés humaines, lequel dynamisme octroie ainsi au monde paysan un aspect sans doute changeant (H. TCHÉKOTÉ, 2015). Dans ce registre, on est en droit de se demander comment les sociétés villageoises traditionnelles, reconnues qu'elles soient par la permanence des traditions ancestrales réussissent-elles à s'accommoder aux réalités nouvelles qu'apporte le contact avec le monde urbain et par rebondissement les innovations issues des transformations de la société globalisée (H. MÉSINI, 2004). Bien plus encore, au regard du caractère particulièrement agricole du paysannat africain et camerounais dominé par le travail de la terre, il se dégage une autre préoccupation à enjeu socio-anthropologique qui interpelle l'adaptation des valeurs d'un héritage ancien à ceux qu'apporte le monde moderne en mutation. Ainsi, il faut convenir avec J-M ÉLA (1983), que, «si l'on veut comprendre la société africaine dans son devenir, avec ses tensions et ses conflits internes, il n'est plus possible de laisser hors du champ d'études les multiples problèmes posés par l'essor urbain » et son influence sur la dynamique rurale. De cet enjeu, il convient de souligner qu'en Afrique, les faits marquants résident dans son passé pré et post colonial d'observance des valeurs occidentales et dans l'influence que lui imprime les interconnexions culturelles nées du processus de mondialisation (J-M ÉLA, 1990). Celui-là, plus que tout autre phénomène, accentue les transmutations qui touchent les paysanneries camerounaises en l'occurrence. Fort de ce constat, il s'agit de cerner les contours de la nouvelle identité paysanne soumise aux vicissitudes que connait la société villageoise, ce à partir des mécanismes déployés pour s'arrimer à un monde sans cesse changeant.

Cette étude est un regard original porté sur les rapports villescampagnes au Cameroun en particulier et par extension en Afrique. Elle met donc en exergue les nouvelles dynamiques des connexions entre l'urbain et le rural, ainsi que les influences de ces connexions sur les transformations des deux espaces respectifs.

\section{Méthodologie et cadrage théorique}

L'étude repose sur une approche qualitative. Elle procède par des observations directes en milieux urbain et rural camerounais. La collecte des données a été faite au moyen d'entretiens structurés réalisés auprès de vingt individus dont dix paysans issus des campagnes représentant les dix régions 
que compte le pays, et dix citadins ayant préalablement vécu en campagne et représentatifs des aires culturelles de ce même pays. En procédant à une analyse des récits d'acteurs, cette réflexion a été bâtie afin de rendre compte de la connexion villes-campagnes. sélectionnés.

C'est grâce à un échantillonnage aléatoire que les participants ont été

Plus précisément, l'échantillon est constitué de dix paysans ressortissants des localités rurales des dix régions camerounaises et dix citadins ayant préalablement été socialisés dans différents contextes culturels paysans et qui vivent désormais en ville.

Une analyse lexico-sémantique permet de mettre en évidence les sens que les acteurs donnent à leurs pratiques en milieux urbains et ruraux, ainsi qu'à leurs différentes stratégies d'adaptations dans un contexte de transformation continue de leurs espaces de vie; ceci à partir d'une grille d'analyse basée sur l'approche dynamiste.

L'extrapolation sur l'ensemble du continent africain résulte des caractéristiques du Cameroun qui est admis comme une Afrique en miniature $\mathrm{du}$ fait de sa diversité environnementale et humaine concordant quasiment avec les aires géographiques et culturelles du continent africain.

\subsection{L'identité du paysan africain}

$\mathrm{Au}$ XIXe siècle, «le terme paysan renvoie-t-il les populations agricoles à leur propre réalité ? » Telle est la préoccupation que posait R. HUBSCHER (1997 : 1), dans sa tentative d'apporter une approche définitionnelle novatrice de l'identité paysanne. En pensant la culture et la profession comme les principaux facteurs constitutifs de l'identité, cet auteur pense que le paysan n'est pas cet individu atemporel doté d'une série d'attributs identitaires construits et véhiculés par l'imaginaire social, mais qu'il y'a « une dynamique et une mobilité identitaire faisant varier le sentiment d'appartenance selon le changement de position et de statut à l'intérieur de la société rurale, l'âge, la diversité des activités exercées, l'insertion dans tel ou tel réseau, l'ancrage territorial, autant de signes de reconnaissance et de référence bien plus évocateurs pour l'homme de la terre que la notion abstraite de paysan » (idem : 8).

Il pose de ce fait, la nécessité de saisir l'identité paysanne à partir des logiques d'actions dans une société rurale elle-même dynamique. Dans le même sens, R. MER (2005), qui lui voit une crise de l'identité paysanne exprime lui aussi la nécessité de revenir au fondement pour reconstruire l'identité du paysan à partir de sa fonction sociale, celle de pourvoyeur des biens agricoles. Pour lui face aux nouvelles logiques qu'apportent le capitalisme, la modernité et l'évolution technologique, il faut en permanence 
reconstruire le sens du métier d'agriculteur que revêt le paysan afin que celuici préserve son identité.

\section{2. les dynamiques paysannes : entre continuité et rupture}

Envisager les dynamiques rurales comme étant des faits nouveaux est une entreprise prétentieuse. En effet, comme toutes les sociétés humaines, le monde rural est depuis bien longtemps traversé par des dynamiques. Lesquelles sont conséquentes soit aux innovations internes paysannes, soit aux influences externes consécutives à leurs rapports avec les centres urbains et par extension le reste du monde.

En effet, d'après J-M ÉLA (1998 : 145), « pour repérer les lieux où les sociétés africaines sont mises en demeure d'inventer les modes d'adaptation au contraintes d'une crise durable, il est nécessaire de porter un nouveau regard sur le rural qui constitue l'espace d'encrage des communauté villageoises ». Il relève ainsi la nécessité de penser l'Afrique rurale à partir des «dynamiques qui la traverse, les acteurs qui orientent sa vie dans le monde d'aujourd'hui » (ibidem). Ainsi, pour l'auteur les transmutations que connait le milieu rural africain lui confère un nouveau visage qui impose qu'on le regarde différemment aujourd'hui plus que hier.

Pour sa part, M. LESOURD (1997 : 364), relève que même-ci elles sont récentes, les mutations paysannes dans les pays du Sud ne sont pas moins importantes que celles des pays du Nord. Pour lui, « l'espace rural africain est le récepteurs de multiples influences externes, en même temps qu'il exprime les impulsions données par les sociétés locales ». À partir du déploiement qu'il reconnait aux acteurs du monde rural, il en arrive à conclure qu'au-delà des contraintes qui l'accablent, la ruralité africaine parvient tant bien que mal à s'adapter avec « souplesse » aux changements que lui imposent ses rapports avec le reste du monde. Il reconnait cependant que dans son élan de dynamisme, la société rurale doit se doter des capacités à pouvoir s'arrimer à l'évolution technique et technologique ; condition essentielle de sa survie.

\subsection{Les nouvelles connexions villes-campagnes}

Les débats relatifs aux rapports villes-campagnes sont anciens. Cependant, du fait des nombreuses dynamiques que connaissent ces différents espaces consécutivement aux mutations de la biodiversité et démographiques, les débats sur la connexion entre la ville et la campagne et leurs fonctions sociales entrainent depuis, des prises de positions contrastées. D’après E. MATHEISEN (2015:4), « avec l'urbanisation exacerbée par l'exode rural, on assiste à la réduction et à la disparition de plusieurs zones rurales ». Il se pose à ce niveau la question de l'influence de la périurbanisation sauvage sur les capacités de production des zones rurales et sur celles de l'approvisionnement des villes en denrées agricoles. C'est la raison pour laquelle, il pense que la 
fourniture de denrées alimentaires provenant de l'agriculture constitue le premier lien entre les villes et les campagnes. Il estime aussi qu'avec l'émergence et la croissance continue des villes, les faits marquants d'actualité restent la forte migration des paysans vers les zones urbaines et l'urbanisation non viable. Pourtant, les approches classiques en faveur de la séparation entre le monde rural et le monde urbain négligent souvent leurs relations et interactions.

Pour H. MAINET et E. KIHONGUE (2015 :2), «Au-delà des définitions statistiques ou officielles des villes petites et moyennes qui varient d'un pays à l'autre et entre auteurs, ce qui importe, c'est bien leur positionnement d'interface dans un espace relationnel qui articule dynamiques urbaines de rang supérieur et dynamiques rurales ». Leur intérêt porte donc sur la place des villes dites intermédiaires dans les rapports villes-campagnes. Ils pensent que les villes intermédiaires par leurs structures jouent un rôle d'interface entre les campagnes et les grandes métropoles. Elles favorisent notamment le développement des petites économies et les transitions migratoires entre les zones rurales et les zones urbaines.

Selon E. N. HOUNGBO (2015), la campagne et la ville sont deux facettes d'une même réalité spatiale et socio-temporelle mue par l'accroissement de la population. Elles ne s'opposent donc pas. De son point de vue, dans la connexion villes-campagnes, tout se passe comme si « la ville chasse la campagne ».Pourtant, la coexistence du rural et de l'urbain reste indispensable pour les populations. C'est ce qui justifie l'exercice de l'agriculture périurbaine, laquelle traduit une juxtaposition du rural et de l'urbain.

\section{Résultats et discussions}

Les transformations du paysannat africain, tout comme la configuration des connexions entre les villes et les campagnes du continent est impulsée par la dynamique dérivée du vaste mouvement d'interconnexion planétaire qu'est la mondialisation. Ces transformations sont donc assujetties à des logiques qui opèrent continuellement entre résistance et fléchissement et entre continuité et rupture, faisant des villes et des campagnes en Afrique, des continuums, soutenus par des modes de vie inter changeants de leurs populations, et surtout l'interdépendance et la complémentarité qui les caractérisent.

Toutefois, il y a lieu de toujours se demander dans une telle situation, c'est-à-dire, au regard de la nouvelle configuration des rapports villescampagnes, si leurs imbrications telles qu'elles se présentent en Afrique et au Cameroun, ne dissimulent pas les différences qui existent entre elles et par extension, la prééminence de l'une sur l'autre. 


\subsection{L'Afrique paysanne, un espace de survivance des anciennes pratiques culturelles et culturales}

À tort ou à raison, l'Afrique est perçue de l'extérieur comme un continent où les faits traditionnels ou anciens sont pérennes et remarquables. Dans certaines circonstances, cette conception peut sans doute se confirmer et dans d'autres, elle ne donne point à être prise sans aucun discernement. Aucun peuple au monde n'existe sans tenir compte de son passé, fusse-il ancestral, les valeurs qui continuent de marquer son existence. L'Afrique traditionnelle, notamment le paysannat au sud du Sahara, caractérisé qu'il soit par la prépondérance du travail de la terre, reste encore pour une part considérable, caractérisé par la persistance des valeurs d'une hérédité sociale ancienne. En se faisant perceptible dans l'ensemble des institutions sociales des peuples d'Afrique, la permanence des traditions d'héritage ancestral illustre à quel point le présent d'une société rend toujours compte de son passé. L'univers du paysan africain et singulièrement camerounais, n'échappe pas à cette logique tant il est le témoin privilégié du passé des peuples modernes qui accordent encore de l'importance à leurs acquis ancestraux. En témoignent les pratiques funéraires qui dans leur ensemble s'opèrent encore dans le cadre des rites anciens, bien celles-ci à défaut de subir des aménagements sont progressivement délaissées par les générations modernisées. C'est ce que semble reconnaitre ce paysan lorsqu'il affirme : «c'est vrai que les choses changent petit à petit, mais nos coutumes sont encore là. Surtout ici à l'Ouest, un week-end ne peut pas passer sans que vous ne voyiez les gens venir au village enterrer leurs morts; ils viennent de partout. [...] pour les rites c'est vrai qu'on ne fait plus tout les rites, mais il y'a beaucoup de choses qu'on fait encore ».

Le village est donc à la fois le reflet de ce passé qui pour une part non négligeable imprègne et inspire le temps moderne. Loin d'être des espaces où s'expriment exclusivement les faits d'une certaine ancienneté, les campagnes africaines traduisent aussi l'existence des réalités nouvelles reçues des transformations de la société mondiale et conséquemment, des milieux urbains voisins. Toutefois, plus que dans les villes, ces faits anciens marquent le quotidien des ruraux. Les zones rurales africaines sont donc les lieux où la tradition des peuples est vécue de façon intense. Les croyances anciennes diverses y trouvent leurs points d'ancrage et donnent à voir en ces villages africains, des lieux qui gardent ou qui préservent les legs ancestraux.

La caractéristique agricole et particulièrement culturale de l'Afrique paysanne témoigne de la permanence des pratiques et techniques anciennes qui peinent à laisser du terrain aux innovations telles que la mécanisation agricole, le recours aux semences améliorées entre autres. L'adoption par le cultivateur des techniques qui inscrivent le travail de la terre au Cameroun dans une logique de durabilité profitable aussi bien aux populations locales 
qu'à d'autres par extension, hors de ce pays reste encore embryonnaire. Comme l'explique ce cultivateur de l'Est du pays, «ici à Mboma, nous travaillons la terre ici nous-mêmes. Mais ce que nous produisons c'est d'abord pour manger entre nous. Après l'autre, on peut envoyer en ville ». Certes cet état de fait peut s'expliquer par la difficulté qu'ont les paysans locaux à se procurer les moyens nécessaires à la pratique d'une agriculture de type intensif et commercial, mais il ne faut pas perdre de vue que ce sont ces mêmes agriculteurs qui tiennent toujours à haute estime, leur tradition culturale et ne se prédisposent pas toujours à les remplacer ou à les concilier aux nouveautés qui s'offrent à eux.

\subsection{De l'incursion coloniale à la tendance à l'hégémonie des valeurs occidentales sur le paysage traditionnel africain}

L'entreprise coloniale dite de civilisation des peuples indigènes ne s'est pas opérée en Afrique sans y laisser des traces parfois inaltérables sur le quotidien des peuples. En effet, comme le constatait il y à longtemps D. MBUNDA (1983), il semble qu'il n'existerait plus aujourd'hui de groupe ethnique traditionnel véritablement authentique, qui n'aurait plus ou moins été influencé par la modernité. L'acculturation des Noirs s'est faite de manière irréversible à telle enseigne que les valeurs endogènes sont tantôt conciliées à celles transposées par le colon, tantôt délaissées au profit des éléments culturels d'emprunt européens. Un regard inattentif porté sur le quotidien des peuples africains et camerounais en l'occurrence donnerait à conjecturer que ce deuxième versant de l'acculturation est plus apparent. Pourtant, avec un recul objectivant, force est de constater que les Africains n'ont pas délaissé totalement leurs traditions ; ils les vivent dans une sorte de continuité qui ne disqualifie pas non plus entièrement les acquis nouveaux issus du contact avec la culture européenne. Plusieurs faits soutiennent cette idée. Dans le Grand Nord-Cameroun par exemple, les chefferies traditionnelles constituent encore des instances privilégiées de gestion des conflits. C'est ce que nous explique le Lamido ${ }^{6}$ de Mango, un village de l'arrondissement de Poli, en périphérie de Garoua, le chef-lieu de la région du Nord. « Là où nous sommes, nous sommes tous ensemble quand vous avez un problème vous allez rencontrer le Djaoro ${ }^{7}$, le Djaoro va voir le Lamido si ça le dépasse. La langue et les dents ne se séparent jamais ». Dans la région du sud les paysans dans leur ensemble, bien que soumis à l'influence des grands projets structurants vivent encore en conformité avec leurs valeurs originelles. À Lolabé et Lendi, des localités rurales à proximité du port en eaux profonde de Kribi, les ruraux sont encore attachés à l'idée que leur vie est intrinsèquement liée à leur espace de vie.

\footnotetext{
${ }^{6}$ Chef traditionnel de premier dégré

${ }^{7}$ Chef traditionnel de troisième degrés
} 
Comme l'affirme cette paysanne «notre terre c'est tout pour nous. Ici à Lolabé, si on nous la prend on aura plus rien. C'est ce que nous savons faire. [...] mais depuis qu'ils sont venus ici avec le port là, les choses sont en train de changer ». Dans nombre de villages à l'Ouest-Cameroun, le recours aux logiques magico-religieuses est encore récurent dans les processus de gestion des affaires courantes de la cité. L'un des informateurs rencontré à Bati, un village périphérique de Galim dans les Banboutos nous rapporte que : «ici chez nous il y'a encore les endroits où il y'a la maison du tonnerre. Quand tu as un problème avec quelqu'un, si tu sais que tu as raison, tu vas voir celui qui gère la maison du tonnerre, il va envoyer la foudre sur celui qui te dérange. Même les gens de la ville connaissent ça. Nous on voit que aller porter plainte au commissariat où au parquet c'est un peu compliqué ».

D'une part, ces récits traduisent l'attachement que les paysans camerounais dans l'ensemble ont vis-à-vis de leurs cadres de vie traditionnels et des valeurs qu'ils tiennent à haute estime, ainsi que la gêne qu'ils ressentent face aux nouveautés que leur imposent l'urbanisation et son corollaire la modernité.

D'autre part, ils évoquent l'identité hybride des citadins qui, quoiqu'influencés par la modernité, opèrent en situation de nécessité, des recours aux pratiques admises comme villageoises.

\subsubsection{Quand les patterns culturels « idéaux » assiègent les villes et villages africains... \\ L'implantation de la culture occidentale s'est accélérée à travers} l'entreprise coloniale qui a ainsi permis une large diffusion de cette dernière en Afrique. Progressivement, le modèle importé s'est improvisé aux yeux de très nombreux Africains comme la valeur par excellence qu'il faut adopter pour connaître le bien-être auquel tout le monde aspire. C'est ce qu'ESSÉAMOUZOU (2009 : 20), appelle «le développement, la chose du blanc »,en allusion à la couleur de peau, à la race du porteur du modèle considéré comme «idéal » de culture qu'il faut au monde pour s'améliorer. Cela dit, le passé du continent noir a longtemps souffert de préjugés au point de donner «l'image d'un espace de tout temps arriéré, où la science, la technologie, la réflexion philosophique... étaient inconnues des autochtones avant le contact avec les Arabes et les Européens » Cheikh M'BACKÉ DIOP, (2011 : 309). Cette image a amplement été relayée par de nombreux africains, non seulement dans les villes, mais désormais aussi dans les villages jusquelà considérés comme les forteresses où les valeurs africaines devraient avoir un droit de cité solide et inébranlable.

Bien que les villes africaines soient aujourd'hui encore plus que les milieux ruraux affectées par le contact avec les civilisations étrangères, c'està-dire celles extérieures au continent noir, il faut remarquer que ces dernières 
ont gagné du terrain et tendent à s'imposer comme des modèles par excellence qui devraient gouverner le vécu des peuples africains. La civilisation occidentale notamment, imprègne la vie de nombreux africains implantés dans les milieux urbains et traduit de ce fait ce qu'il convient d'appeler l'occidentalisation des villes africaines. Celle-là tient sur la permanence du mode de vie occidental érigé en modèle et tend à reléguer au second plan, les anciens acquis culturels endogènes. Toutefois, l'idéalisation du mode de vie «américano-européen » ne disqualifie point la présence des cultures locales dans les métropoles du continent noir. Les citadins africains, au même titre que leurs congénères des milieux ruraux sont donc confrontés à une dualité, pour ne pas verser dans les spécificités à une pluralité de modes de vie au point où leur quotidien reste jonché de comportements et pratiques puisant tantôt dans une source de production culturelle locale, c'est-à-dire africaine, tantôt dans la fontaine qui diffuse des cultures de provenances diverses par l'entremise des phénomènes urbain et mondial. On peut donc conclure dans ce contexte à la «dynamique » et la «mobilité » identitaire envisagées par R. HUBSCHER (1997), tout en précisant dans sa continuité qu'elles n'influencent pas que le paysan, mais aussi le citadin. Du coup, le type africain se trouve dans une sorte d'embarras quant à l'adoption de telle ou telle autre mesure face à des situations qui l'interpellent et lui propose facultativement d'opérer un choix circonstanciel. Ainsi, alors que le paysan est hésitant sur l'adoption d'une technique ou d'une pratique culturale moderne qui lui offre de nouvelles perspectives par exemple, le citadin reste indécis sur l'appartenance de son mode de vie au terroir africain ou européen. Il vit certes en Afrique, mais son quotidien tend à ressembler plus, et ce de façon continue et irréversible, à ce qu'il perçoit ailleurs à travers les médias de masse que sont la télévision en premier lieu, et internet ensuite. Ces cas de figures illustrent la tension qui tiraille l'Africain entre sa tradition et la modernité et se répercutent dans les profondeurs des institutions socioculturelles. Le contact de l'Afrique avec l'Occident donne à penser qu'il s'agit là de deux mondes, l'un étant aux antipodes de l'autre, avec l'un, l'africain de négativité et l'autre, l'européen porteur d'inventivité propice au développement. Cet état de fait n'est pas sans incidence sur les mentalités des africains qui se trouvent aussi bien en ville que dans les villages écartelés entre deux réalités qui se côtoient pourtant.

\subsubsection{L'embarras face aux nouvelles valeurs à larges capacités de diffusion s'improvise}

Alors que les transformations que subissent les sociétés villageoises se poursuivent de façon accélérée sous l'impulsion de la mondialisation et que les cultures se transportent à travers la planète indépendamment de leurs différents groupes sociaux d'élaboration, il se révèle une présence accrue de valeurs culturelles importées de provenance diverses dans l'univers du paysan 
en Afrique. Le constat fait état d'une existence fort remarquable des composantes culturelles de la civilisation occidentale qui ne cesse de gagner $\mathrm{du}$ terrain aussi bien en milieux urbains qu'en zones rurales africaines. En interrogeant l'histoire de ces contrées, l'on arrive à la compréhension qui voudrait que les grandes explorations et le colonialisme aient été les faits marquant de la venue de l'homme et de la culture occidentale en Afrique.

Aujourd'hui pourtant, ces pages de l'histoire du continent noir sont dépassés et la civilisation portée par les explorateurs et colons continue d'imprimer son influence sur le quotidien des africains au point de tendre à supplanter ou à dominer sur les héritages traditionnels ancestraux. Les mouvements continus des hommes et surtout aujourd'hui encore, les nouvelles technologies, favorisent le transport des éléments culturels à travers le monde. La multiplication des échanges mondiaux ouvre une piste où les cultures sont en rapport de compétition les unes avec les autres. Dans un tel contexte de compétition, certaines traditions perdent progressivement du terrain au profit de celles à large capacité de diffusion. Ainsi, le paysannat africain se trouve lui aussi transformé en terrain de compétition culturelle et exprime de surcroît la confusion qui règne dans les esprits partagés entre le matraquage culturel idéalisant les valeurs nouvelles et la pérennisation des savoirs endogènes antiques. De plus en plus donc, le modèle agricole incarné par la société mondiale voudrait que le travail de la terre soit tourné vers la durabilité afin que l'être humain puisse convenablement satisfaire ses besoins de nutrition sans doute nécessaire à son épanouissement. À cet effet, l'expérimentation des progrès scientifiques et techniques dans le domaine agricole, devient une alternative qui s'offre aux paysans africains encore tenus par l'attachement aux anciennes techniques et pratiques culturales qui n'augurent pas toujours de meilleurs rendements agricoles. Ce qu'il faut retenir dans une situation d'indécision comme celle-là, c'est que l'agriculture en Afrique, qu'elle soit commerciale ou familiale, doit s'arrimer aux contraintes de la demande grandissante des biens alimentaires en adoptant les mesures les plus appropriées pour combler les aspirations de bien-être qu'ont les hommes. Il faut admettre que ce processus est enclenché depuis quelques décennies, mais il reste encore dans sa phase de balbutiement dans la plupart de la paysannerie camerounaise. Des efforts sont à fournir pour qu'il soit effectif, car l'attachement aux traditions, même les moins adaptées aux nouvelles réalités, continue de prévaloir.

\subsection{Le processus mondial et les connexions « villes-et-villages» en Afrique}

Le monde se transforme, l'Afrique s'urbanise et les milieux paysans africains ne sont pas à la traine de ce dynamisme ambiant. La transition urbaine dans ce contexte représente une opportunité, mais implique aussi des 
défis relatifs à la gestion des ressources (C. KESSIDES, 2006). Sous l'impulsion de la modernité que transporte la mondialisation, les réalités africaines connaissent des changements et les Africains eux-mêmes en sont parties prenantes car, ils voient leur environnement de vie s'en accommoder. Les villes et les campagnes ont longtemps été considérées comme deux types de sociétés fortement différentiées où le premier serait le centre et le second la périphérie, l'un hétérogène et l'autre homogène. En bref, la ville et le village ont toujours été perçus comme étant aux antipodes l'un de l'autre. Pourtant, au regard de la configuration actuelle des rapports villes et campagnes, des questionnements naissent de la pensée.

\subsubsection{Les campagnes administrées à la fois localement et à partir des villes}

Les milieux ruraux aujourd'hui plus qu'hier, sont des espaces de prise de décision pour le devenir des villageois. C'est au village en Afrique et au Cameroun précisément, que les autorités religieuses et les chefs traditionnels disposent des pouvoirs au point de s'arroger le droit de veiller sur le bien-être de leurs communautés. Comme l'affirme ce gardien de la tradition à $\mathrm{Mbakaou}^{8}$ «je suis le représentant de l'administration ici. C'est moi le gardien des traditions et du bien-être de ma population. Donc quand il y'a une situation ici, on se réfère d'abord à moi et moi je rends compte à ma hiérarchie ». Ces autorités disposent aux yeux des populations, d'une réputation à eux conférée par la tradition toujours présente dans le quotidien des hommes. La situation telle qu'elle se présente actuellement, conforte l'idée selon laquelle « la ruralité ne se définit pas seulement par les activités productives liées à la terre et aux systèmes d'exploitation de la nature » (M. LESOURD, 1997).

Autrefois «détachés » des centres urbains, les villages africains ne bénéficiaient qu'occasionnellement de l'attention de l'administration publique alors fortement concentrée et centralisée. De nos jours, les politiques étatiques de rapprochement des administrés de l'administration ont accordé une marge d'agissements aux populations rurales qui sont désormais devenues ellesmêmes, les maîtres de leurs propres desseins. Les villages sont administrés par des élus locaux ayant moins de connexions de subordination avec les acteurs politiques et administratifs des grands centres urbains. Il faut reconnaître pour cela que les transformations de l'espace rural en sont un résultat conséquent. Elles se font de plus en plus visibles avec désormais le nombre croissant de ses habitants de provenances diverses, la grande variété des activités qui y sont exercées, les aménagements publics etc. Bref elles sont en continuelles rénovations. Toutefois, il convient de ne pas perdre de vue que la ville, en tant

${ }^{8}$ Petit village de l'Adamaoua, périphérique à Meiganga 
que vitrine de la modernité, inspire les villages. Plus que cela, elle donne à être la locomotive pour les villages implantés dans sa zone d'influence.

Les villes ont toujours été par leurs activités, les centres de prises de décisions en ce qui concerne le fonctionnement d'un pays. À cet effet, elles assurent le rôle de commande d'autres villes et par rebondissement des milieux ruraux. Parce qu'elle est à la fois le centre des pouvoirs administratifs et économiques, le milieu de production des biens et des services, le carrefour de la communication, le pôle d'échange des marchandises et des capitaux etc., la ville imprime par ces caractéristiques de centralisation, son influence audelà de la stricte étendue qu'elle recoupe. Subséquemment, autant elle est importante en superficie et en population, autant sont nombreuses ses fonctions et son influence sur les villages avoisinants. De même, les grandes villes jouent le rôle de direction des affaires alors que les milieux villageois sous leur dépendance assurent des rôles secondaires. À partir des villes se déploient des politiques dont les effets, en se répercutant montrent à quel point les grandes décisions concernant la marche d'une Nation se prennent dans ses villes phares ; celles-là sont à la fois les sièges des institutions publiques et les portes-flambeaux de la modernité parce qu'étant plus marquées par des nouveautés.

\subsubsection{Les villes-et-villages sans frontières : agencements routiers, flux des personnes et biens}

Dans les centres urbains en Afrique, on retrouve de nombreuses banlieues dont l'extension est facilitée par le développement des réseaux routiers qui les traversent. Les espaces ruraux voisins sont progressivement phagocytés par les villes et transformés par la périurbanisation. C'est le cas à Ayos dans l'Est du pays où un citadin nous rapporte « avant il n'y avait rien ici, c'était le village et le champ. Mais quand on a dit à nos parents que la route va passer, on les a envoyés ici. Aujourd'hui les gens ont construit un peu partout c'est même déjà la ville ». Les routes relient non seulement les quartiers urbains mais aussi la ville à ses banlieues au point de donner à penser qu'il n'y aurait pas de rupture entre une cité et ses zones périphériques. Cette connexion visible à travers le réseau routier étend pourtant ses embranchements jusqu'aux villes et villages voisins, faisant ainsi des agglomérations distantes l'une de l'autre, et de celles qui se côtoient, un grand ensemble unifié où les limites non conventionnelles sont difficiles à circonscrire. De plus en plus, rares sont les localités, qu'elles soient urbanisées ou non, qui n'aient pas de connexion routière avec d'autres du même contexte environnemental ne serait-ce que.

Les villages, les campagnes, ou encore les paysanneries africaines ne présentent plus l'image absolue des zones reculées ne disposant d'aucune jonction « routière » avec les milieux urbains. Cette configuration de la 
relation entre les villes et les campagnes contraste avec la conception précoloniale où le phénomène de l'urbanisation des États africains était encore élémentaire. L'urbanisation de l'Afrique se poursuit rapidement et elle s'accompagne sans doute de l'augmentation des populations urbaines. Ce qui veut dire que, comme l'indique E. N. HOUNGBO (2015), la croissance des villes rime avec l'augmentation des populations, laquelle augmentation reste fortement tributaire de l'exode rural qui en est un pourvoyeur de nouveaux citadins. Ce qui ne signifie pas que les populations urbaines proviennent essentiellement des milieux ruraux paysans. Elles sont aussi issues des naissances localement enregistrées et des migrations transfrontalières facilitées par la mondialisation. Toutefois il convient de ne pas ignorer ou minimiser ce qui se rapporte à l'exode urbain, ce départ en sens inverse des personnes qui désertent plutôt les métropoles pour s'installer dans les villages. $\mathrm{Au}$ Cameroun, ce phénomène lui aussi de plus en plus grandissant témoigne de l'échec de la ville à satisfaire ses occupants ou encore de l'attraction que suscitent désormais les milieux ruraux et des faveurs qu'ils octroient à leurs locataires. Pour certains, « en ville tout est chers et il y’a trop de problèmes »; pour d'autres, « le village est un cadre idéal pour prendre du recul avec toute la pollution de la ville, les bruits, et se reposer, respirer la bonne aire du village ».

La ville et la campagne se complètent dans la perspective de la satisfaction des aspirations des personnes qui y séjournent. Cependant, il faut le souligner, un regard attentif porté sur la composante humaine que ce soit en ville ou dans les milieux villageois, laisse percevoir une hétérogénéité des populations, contrairement à une conception ancienne qui voudrait que seules les mégalopoles connaissent de fortes mixités sociales. En matière de composantes sociales, les villes et les villages africains ont tendance aujourd'hui, à se ressembler. Du villageois, on en trouve en ville et du citadin également, on en trouve au village. Autant les populations d'une contrée rurale qui se retrouvent en ville procèdent par recomposition ethno-tribale pour s'identifier et mieux intégrer la sphère urbaine, autant les citadins partis de la même ville se construisent une communauté urbaine transposée en campagne. Ce dernier cas de figure se comprend encore plus quand on admet que la ville d'aujourd'hui a certainement été à un moment donné de l'histoire, le « village » d'une ou de quelques communautés, le village étant entendu ici comme le milieu d'origine et d'identification de certaines composantes tribales ou ethniques.

Par ailleurs, la disparité entre les zones urbaines et les milieux paysans réside dans les caractéristiques communément reconnues aux premières d'être des pourvoyeurs de produits de manufacture alors que les seconds se distinguent dans la fourniture des aliments de provenance agricole. Nonobstant, ces différentes considérations allouées aux villes et aux 
campagnes camerounaises en l'occurrence, tendent à tomber en désuétude car, de plus en plus, les campagnes sont les espaces d'implantation des industries transformatrices des biens issus des travaux paysans. La prolifération en ville de l'économie informelle, pourtant principale caractéristique des marchés ruraux, atteste certainement de la transposition des habitudes villageoises en zones urbaines, et par ricochet, témoigne du souci qu'ont les citadins de contourner les difficultés de survie que leur impose le séjour urbain, même s'il faut y pratiquer des activités traditionnellement reconnues aux zones rurales.

Ce qu'il faut noter des réalités autour des connexions entre les villes et campagnes en Afrique et au Cameroun, c'est aussi le dynamisme qui les anime et leur confère des visages à la fois anciens et nouveaux adoptés sous l'effet des mobilités humaines accentuées dans les sens aller et retour.

\subsection{L'identité du paysan africain mondialisé}

L'espace rural africain d'aujourd'hui, plus que celui d'autrefois, est en même temps unique et diversifié.

Dans un sens, il présente le visage d'une Afrique traditionnelle marquée par la continuité culturelle qui transporte avec elle les valeurs anciennes.

Dans un autre sens, il rend compte de la rupture qui traduit l'incursion des faits de modernité appelés soit à dépasser les antiques pratiques encore en cours, soit à coexister avec elles.

À partir de là, il devient difficile de définir l'identité de l'africain rural partagé entre la tradition et la modernité dont les éléments caractéristiques proviennent généralement de la ville.

\subsubsection{Le quidam mondialisé du paysannat africain entre tradition et modernité}

Une culture vivante est celle qui interagit avec les autres; les langues, les religions, les échanges économiques et culturels, les migrations en tant que résultantes de la mondialisation, sont à l'origine des transformations que connaissent les cultures à travers le monde. Ces dernières sont donc soumises à des mutations au point où l'on est en droit de se demander si le processus continu de la mondialisation qui ne saurait s'arrêter constitue une menace pour la diversité culturelle de notre humanité. Ce questionnement est d'autant valable alors que, comme on le sait, de plus en plus les peuples économiquement dominés dénoncent le phagocytage, c'est-à-dire l'absorption en vue d'une neutralisation de leurs valeurs culturelles et surtout la prépondérance du mode de vie américain et partant, occidental en général. Visà-vis de ces états des faits, on se demanderait alors si c'est simplement l'hégémonie de la culture occidentale et la crainte d'une éventuelle occidentalisation à venir du monde qui pousse les peuples à des revendications 
identitaires, à des replis communautaires tels qu'ils sont visibles dans bon nombre de régions du monde.

\subsubsection{L'incontournable pluralisme identitaire des villes africaines}

Chaque individu ressent la nécessité d'affirmer son identité pour en faire son repère ou sa boussole dans les interactions sociales auxquelles il est quotidiennement associé. Telle est l'une des caractéristiques propre de l'humain. Pourtant, à partir de ces interactions, il est également confronté à une autre nécessité, celle d'emprunter des éléments d'autres identités voisines qu'il doit intégrer dans la sienne pour s'améliorer et s'adapter face aux contraintes de la vie. Ce qui le pousse aussitôt à renoncer à une identité réductrice ou à sens unique, hostile à celle de l'autre. D'un point de vue de dimension sociétale, le même phénomène s'opère lorsque les groupes d'individus de provenances diverses se côtoient, car ils ont besoin d'être euxmêmes et de communier avec les autres groupes avec lesquels ils entre en contact. Ainsi, la mise en rapport des éléments d'identités d'origines diverses est une réalité particulièrement courante en milieu urbain.

En tant que lieu de rencontres identitaires, la ville africaine est aussi un espace reflet des changements culturels. Elle constitue une sorte d'interface où s'associent, s'agrègent ou se contredisent les modèles traditionnels porteurs d'héritages diversifiés et des vecteurs de modernisation, fondement d'une culture mondialisée. Ici se côtoient hybridation et métissage des cultures au sens anthropologique du terme.

Dans ces espaces cosmopolites, les discours identitaires ne s'articulent pas forcément et prioritairement en fonction d'affinités linguistiques, même si l'appartenance linguistique peut certes être un pilier important de l'identité.

\section{Au-delà de la connexion ville-campagne}

De plus en plus les villes africaines et les campagnes qui les avoisinent se tiennent et exercent leur influence, les unes sur les autres dans un jeu cyclique de réciprocité continue où il est difficile de remarquer des étapes de transition. Certes le village précède la ville, mais cette dernière accorde son sens au précédent dans la mesure où la conception générale voudrait que le village, la zone rurale, ou la campagne soit considérée comme telle par rapport à la ville qui est communément admise comme son inverse. Aujourd'hui, la connexion entre ces deux entités initialement considérées comme des contraires, permet de rapporter la dualité et la dichotomie en une sorte d'unité continue.

\subsection{La télécommunication en tant que mécanisme de jonction}

Jusqu'en 1999, pour transmettre des nouvelles entre une ville et une zone rurale, il fallait faire recours aux techniques les plus anciennes qui 
consistent à se déplacer soi-même ou à faire déplacer quelqu'un d'un point à un autre en empruntant divers moyens de locomotion parce que la télé densité liée à la téléphonie fixe au Cameroun était très faible. (NGOUDJOU J-H, 2018). Rappelons que le téléphone fixe lui-même voit le jour alors que le modèle de communication télégraphique devenait obsolète devant le progrès technologique. Ainsi, dans les deux premières décennies des années 2000, avec l'avènement de la téléphonie mobile indissociable qu'elle soit d'internet, de nombreux citadins africains, y compris des habitants de campagnes, vont se sentir beaucoup plus proches les uns des autres malgré la distance évidente qui les sépare. Ce qui signifie que l'incursion du téléphone portable est venu effacer virtuellement les frontières physiques entre urbains et ruraux. Désormais, il est plus facile pour les citadins et les ruraux d'entrer en contact à travers la téléphonie mobile soit par les émissions d'appels, soit par l'envoie des communications au moyen des messageries à textes des réseaux sociaux multiples que les africains se sont appropriés. Par-là, la ville et le village se trouvent l'une distante de l'autre de quelques clics du clavier du téléphone. Précisons que « le téléphone mobile n'est qu'un élément du vaste ensemble que constituent les TIC (Technologies de l'Information et de la Communication)aujourd'hui, parmi tous les outils que l'on désigne sous l'appellation de "Nouvelles Technologies", le téléphone portable et l'internet occupent une place assez particulière; ce d'autant plus que ces outils permettent de relier des personnes éloignées, et se présente comme un moyen de résoudre les problèmes inhérents aux obstacles de distances ».(NGOUDJOU J-H, $2018: 35$ ).

En rapprochant les villes et les campagnes, le téléphone portable transcende le strict réseau téléphonique qu'il contribue à construire entre les urbains et les ruraux ; il en appelle à la constitution de réseaux sociaux incluant concurremment les personnes vivant en ville et celles séjournant au milieu rural en un ensemble, cela au travers des entrecroisements favorisés par WhatsApp et Facebook entre autres. De même, les flux de personnes et biens s'en inspirent pour l'intensification des échanges économiques entre les populations des villes et des campagnes. De ce qui précède, et en considération des voies de communication qui les rapprochent aussi, le citadin et le rural sont de moins en moins soumis à des disparités en ce qui concernent la disposition et l'accès aux biens alimentaires par exemple. Cela dit, il devient récurrent qu'un citadin consomme en plus des biens de manufactures issus des industries urbaines ou des exportations que les villes reçoivent en priorité, des biens de provenance agricole produits par les paysans africains. En compensation, les ruraux sont ravitaillés en produits manufacturés tout comme ils consomment ce qu'ils produisent sur place. Ces faits traduisent simplement la nouvelle configuration des relations entre villes et campagnes africaines 
dont le dynamisme réfléchit la transformation de la société en perpétuelle mondialisation.

\subsection{Le phénomène de périurbanisation en Afrique}

La périurbanisation s'entend comme le procédé d'extension de l'urbanisation dans les campagnes environnant les villes. (A. YEMMAFOUO, 2013). En tant que métropolisation, la périurbanisation a pour effet d'exercer des pressions sur la campagne. Ces pressions se manifestent notamment par la reconfiguration des espaces, la dynamique des rapports à l'espace qui se traduit par l'émergence des comportements mercantiles et aussi la fragilisation progressive des identités villageoises au profit des mœurs urbaines ellesmêmes influencées par les valeurs reçues de la mondialisation. Dans une telle logique, la connexion ville-campagne donne à penser certes qu'on converge vers une désintégration du monde rural pour laisser place à un univers de brassage en perpétuel mouvement, mais aussi et surtout, qu'on assiste à un effacement de l'esprit paysan du fait de l'ascendance que la ville prend sur l'espace rural. Cela y va donc de la crise paysanne qui suggère une rupture parfois brusque d'avec les valeurs les plus fondamentales et les spécificités des peuples. Ainsi, l'effet conquérant de la ville viendrait mettre à mal les identités originelles qu'évoque le monde rural. Ces identités transposées dans des mœurs englobant de la ville participent continuellement à la reconfiguration de la pluralité culturelle ambiante des milieux urbains africains.

Dans un sens, et à titre illustratif, il est apparent que les campagnes s'urbanisent à travers la pression résultant de l'accroissement des populations urbaines environnantes qui les pousse par voie de conséquence, à étirer la ville pour absorber ses périphéries rurales.

Dans un autre sens, la ville se « ruralise », non pas dans la perspective de la régression de cette dernière, mais selon le nouveau visage qu'elle adopte. Cela dit, les ruraux désormais devenus citadins à partir de l'urbanisation et de son corollaire, l'absorption de ses villages périphériques, perpétuent leurs habitudes culturelles villageoises qui les caractérisent. Ils ont tendance à reproduire dans les espaces urbanisés et au-delà, dans les centres urbains qui leur sont désormais devenus plus familiers, les économies villageoises telles que l'élevage, la petite agriculture, le commerce ambulant et informel, etc.

\section{Les « grands villages » ou les « villes-villages » du Cameroun}

Au regard de la poussée des villes vers les villages ou du recul des milieux ruraux au profit de l'urbanisation, il est inévitable que les modes de vie connaissent des transformations conséquentes, compte tenu des réalités nouvelles qui s'improvisent dans le quotidien aussi bien des citadins que des ruraux. C'est d'ailleurs pourquoi en Afrique, la culture urbaine est présente en 
campagne, tout comme la culture villageoise est prégnante en ville. De ce fait, il y a au Cameroun par exemple, de «grands villages »ou des «villesvillages »qu'il est difficile de ranger dans l'ordre des villes ou dans celui des campagnes, même si formellement ils le sont dans l'une ou l'autre des catégories.

À titre d'illustrations, dans les agglomérations des trois régions du Grand-Nord du Cameroun par exemple et dans les villes phares d'autres régions où sont installées des communautés musulmanes, il est courant de voir du bétail non accompagné parmi les «citadins » comme s'il s'agissait des scènes de villages, parce que ces communautés sont connues pour l'intérêt qu'elles accordent à la pratique de l'élevage des ruminants. L'un des ressortissants de cette région du pays interrogé à Yaoundé reconnait que : «la plupart des gens quand ils quittent du nord, ils viennent ici avec leurs bétails. Parce que ici on ne peut pas trouver la terre qu'on va cultiver. En dehors de la couture par exemple, ce que nous avons encore et qui nous permet de survivre c'est le bétail, même-ci c'est vrai que l'élevage n'est pas facile ici en ville ».

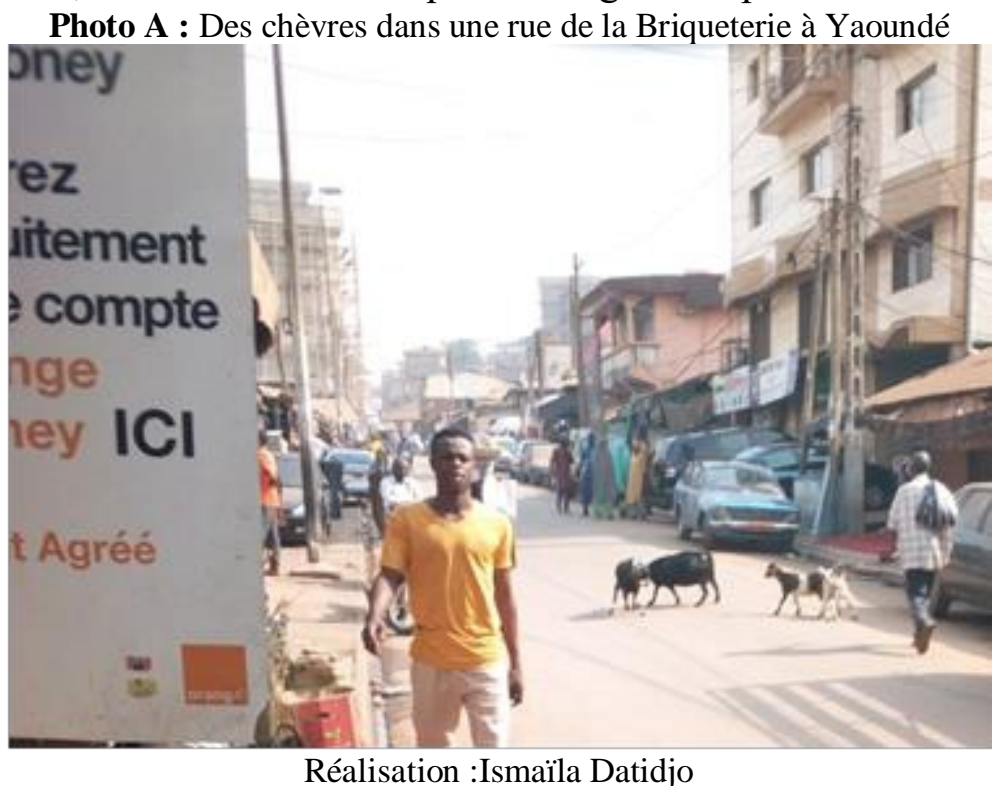

De même, dans les régions de l'ouest, de l'est, du centre et du sud du Cameroun, des cultures longent les allées avec des bananiers à l'ouest notamment, qui donnent l'impression d'être des arbres de rue. En effet comme le dit cette informatrice, " en général nous ici à l'ouest nous vivons toujours avec l'idée que nous somme au village. Chacun a toujours son petit lopin de terre dans sa concession où il fait un peu de champ, c'est difficile de voir quelqu'un ici qui est chez lui et qui n'a pas son petit champ à côté quelque part, parfois même sa porcherie aussi ».L'illustration suivante en témoigne. 
Photo B : Des bananiers à la devanture d'une maison au bord de la chaussée à Dschang

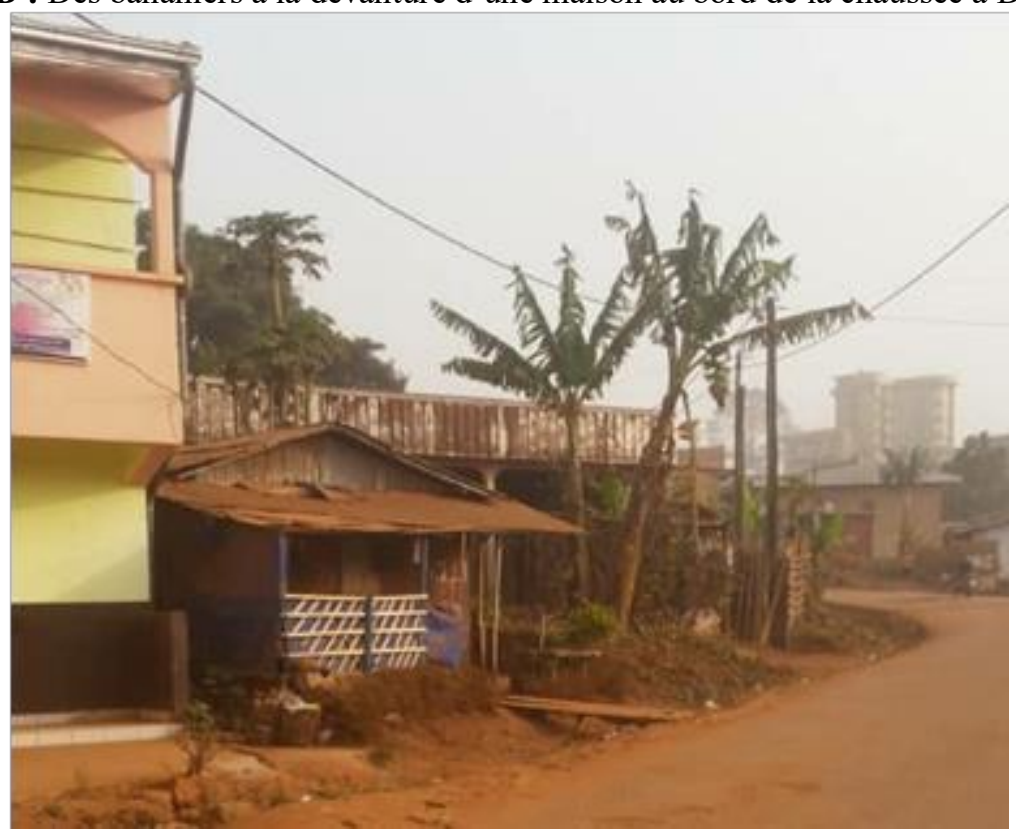

Réalisation : Nana Claudin Karim

Sur la photo B, on voit des plantes de bananiers sur la façade d'une maison construite au bord de la chaussée. Cette pratique est courante à Dschang comme dans l'ensemble des villes de la région de l'Ouest Cameroun. Tout compte fait, ce qui se dégage de ces réalités, c'est la continuité du mode de vie paysan qui peine à laisser du terrain à la culture citadine. Pourtant, celle-ci prévaut, mais en cohabitant avec la pratique des habitudes villageoises qui y sont transposées ou préexistantes malgré la périurbanisation.

\section{Conclusion}

Les rapports entre villes et campagnes sont désormais à sortir des carcans de l'histoire ancienne et de les redéfinir dans le contexte actuel. Il n'est plus question des rapports de subordination ou de domination de l'une face à l'autre. De plus en plus, ces rapports sont ceux d'interdépendance, de complémentarité et de métissage. Désormais, autant les villes ravitaillent les villages en denrées alimentaires d'origine industrielle, autant les campagnes africaines se modernisent et s'industrialisent. En plus, elles approvisionnent les villes voisines et lointaines à partir des bassins de production agricoles qu'elles représentent en biens alimentaires. Pourtant, compte tenu des connexions qui s'opèrent entre citadins et ruraux à travers divers mécanismes que propose la modernité elle-même corollaire de la société globale, il est visible que la ville et la campagne se résument en un ensemble de faits qui se rapportent à la trilogie interdépendance-complémentarité-métissage. Jadis 
lieux de consommation des savoirs faire d'origine urbaine, les campagnes deviennent des foyers de production et de diffusion des biens de consommation destinées aux citadins également. En Afrique, au-delà des limites naturelles entre ville et campagne qui deviennent de moins en moins repérables, la venue des Nouvelles Technologies s'est improvisée comme un facteur d'envergure dans le rapprochement entre campagnards et habitants des villes.

Vouloir dissocier la ville de la campagne en contexte camerounais apparait désormais comme une entreprise difficile dans bien de mesures, tant l'imbrication de l'une dans l'autre donne à ces deux entités des allures de mosaïques. Entre innovations et transports des habitudes culturelles diverses, entre flux de personnes et de biens économiques etc., des villes vers les campagnes, la conception du monde rural comme une espace d'homogénéité laisse progressivement la place à la campagne entendue comme un espace mondialisé, un lieu d'expression des différences.

Entre la rupture qu'impose la modernité aux africains qui adoptent de plus en plus le mode de vie des urbains en délaissant certaines de leurs anciennes habitudes, et la continuité à travers laquelle ils restent attachés à leurs valeurs d'héritage ancien qu'ils perpétuent, les populations africaines développent un mode de vie qui exprime la nouvelle configuration des rapports villes- campagnes dans une Afrique embarquée dans le processus de mondialisation. Il reste qu'au vu des transformations qu' induit cette nouvelle configuration, on convient de s'interroger sur la refondation du mode de gestion de ces rapports ville-campagnes dans un contexte où la décentralisation est admise comme une aubaine pour les Africains.

\section{References :}

1. Cheikh M'BACKÉ, D. (2011). La recherche scientifique et technologique africaine, Paris : Présence africaine.

2. ÉLA, J.-M. (1983). La ville en Afrique noire, Paris : Karthala.

3. ÉLA, J.-M. (1990). Quand l'État pénètre en brousse, les ripostes paysannes à la crise, Paris : Karthala.

4. ELA, J-M (1998). Innovations sociales et renaissance de l'Afrique noire, les défis $d u$ "monde d'en-bas », Harmattan, 426 pages.

5. ELONG, J. G. (2011). L'élite urbaine dans l'espace agricole africain. Exemples camerounais et sénégalais. Paris : L'Harmattan.

6. ESSÉAMOUZOU (2009). L'influence de la culture sur le développement en Afrique noire.

7. HOUNGBO E. N. (2015). «Relations campagne et la ville : deux réalités complémentaires et interdépendantes», in iedafrique.org/IMG/pdf/AGRIDAPE_31-2_juin 2015.pdf 
8. HUBSCHER, R. (1997). «Réflexions sur l'identité paysanne au XIXe siècle : identité réelle ou supposée?», Ruralia (En ligne), mis en ligne le 01 janvier 2003, ournals.openedition.org/ruralia/4.

9. KESSIDES, C. (2006). La transition urbaine en Afrique subsaharienne : impact sur la croissance économique et la réduction de la pauvreté. Washington DC : L'Alliance des villes.

10. LESOURD, M. (1997). «L'archipel rural africain en mouvement » in GASTELLUJ. M.et MARCHAL, J. Y. la ruralité des pays du sud à la fin du $x x^{e}$ siècle (pp :363-378). Paris : édition de l'ORSTOM.

11. MAINET, H. et KIHONGUE, E. (2015). « Les villes secondaires dans les relations villes-campagnes en Afrique de l'Est » in territoire en mouvement, revue de géographie et aménagement. DOI : 10.4000/tem.2938, pp. 1-17

12. MATHEISEN E. (2015). «Relations ville-campagne : vers des systèmes alimentaires intégrés $\gg$, in iedafrique.org/IMG/pdf/AGRIDAPE_31-2_juin 2015.pdf

13. MBUNDA, D. (1983). Valeurs culturelles, traditions et modernité,(online)In http://unesdoc;unesco.org, problème de la culture et des valeurs culturelles dans le monde contemporain, Unesco.

14. MER, R, (2005). «Agriculteurs, paysans et co : crise d'identité et identité des crises » in Quaderni, n56, pp. 101-113.

15. MÉSINI, H. (2004). «Résistances et alternatives rurales à la mondialisation » in études rurales, $\mathrm{N}^{\circ}$ 169-170, (pp. 43-59).

16. NGOUDJOU, J-H. (2018). Technologie de l'information et de la communication et maraîchage de l'Arrondissement de Nkong-Ni (Département de la Menoua) thèse de Doctorat $\mathrm{PhD}$ Université de Ngaoundéré.

17. TCHÉKOTÉ, H. (2015). La ville dans les campagnes de l'Ouest Cameroun: une déconstruction du paysage agricole par l'effet de résidentialité in Les Cahiers du Développement Urbain Durable.

18. VENNETIER, P. (1991). Les villes d'Afrique tropicale, $2^{\mathrm{e}}$ édition, Paris : Masson.

19. YEMMAFOUO, A. (2013). Urbanisation des espaces périurbains en Afrique subsaharienne, pratiques à l'Ouest-Cameroun, Paris : L'Harmattan. 